\title{
FABRICATION OF DENSE FLOW SENSOR ARRAYS ON FLEXIBLE MEMBRANES
}

\author{
N. Izadi ${ }^{l^{*}}$, M. J. de Boer ${ }^{1}$, J. W. Berenschot ${ }^{1}$, R. J. Wiegerink ${ }^{1}$, T. S. J. Lammerink, \\ H. V. Jansen ${ }^{1}$, J. Mogdans ${ }^{2}$ and G. J. M. Krijnen ${ }^{1}$ \\ ${ }^{1}$ Transducers Science \& Technology group, MESA ${ }^{+}$and IMPACT Research Institutes \\ University of Twente, Enschede, The Netherlands \\ ${ }^{2}$ Institut für Zoologie, Universität Bonn, Germany
}

\begin{abstract}
Biological sensory systems often display great performance which inspires engineers to design artificial counterparts. In this paper we report the fabrication of aquatic hair based flow sensors inspired by fish lateral line. Geometrically optimized flexible membranes of SU-8 with integrated electrodes underneath have been realized in a reliable, high yield process. By separating the liquid medium from readout electrodes we decrease squeeze film damping and eliminate electrolysis. The procedure allows dense membrane array fabrication.
\end{abstract}

\section{KEYWORDS}

Biomimetic, Lateral line, Flow sensors, DRIE, SU-8

\section{INTRODUCTION}

The ability of fish to localize pray or predators, avoid obstacles and school, to a great extend depends on a sensory organ called lateral line. This consists of hair shape mechanoreceptors or neuromasts, covered with a jelly like cupola, which are located on the skin or in the canals along the body [1] as shown in figure 1. Fluid motion causes the displacement of cupula coupled with the hair cells inside. The discharge rate of afferent neurons changes in response to displacements of the hair cells [2]. Using this system, fish are able to perceive cupula displacement as small as few nanometers [3]. It is beneficial for study of hydrodynamic interaction in complex, noisy environment and for understanding of physiological behavior of underwater animals, to engineer a flow sensor based on the same principle to reach a high level of robustness and sensitivity.

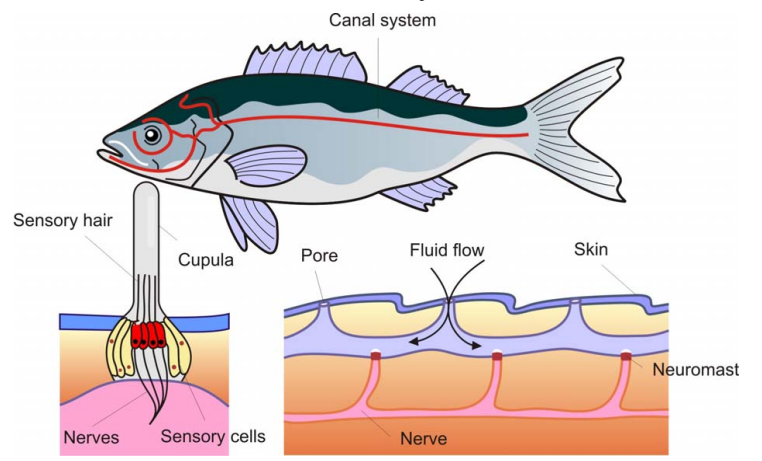

Figure 1: Schematic drawing of fish lateral line. Bottom right shows configuration of canal. Bottom left is a close up of a neuromast. (After Encyclopedia Britannica)
Figure 2 shows a schematic view of the proposed sensor design. This configuration enables differential capacitive sensing upon rotation of the metal electrodes under the membrane due to deflection of a receptive hair by drag force of the fluid flow. The capacitive readout provides high sensitivity, low power consumption and electrostatic softening ability [4]. A fully supported membrane prevents electrode/liquid contact and eliminates the chance of electrolysis and short circuiting. In addition, high damping by squeeze film effect, associated with the presence of fluid in between the electrodes, is avoided. The gap between two counter electrodes can be tuned by the thickness of a sacrificial polysilicon layer to optimize the sensor for the highest sensitivity considering the static downward deflection of the membrane due to pressure difference. An optimized DRIE process to access a sacrificial layer from the backside of the wafer provides a suitable procedure to realize dense arrays of hair sensors. The shape and length of SU-8 hairs can also be optimized for higher drag force and therefore more mechanical deformation of the membrane which leads to higher device sensitivity.

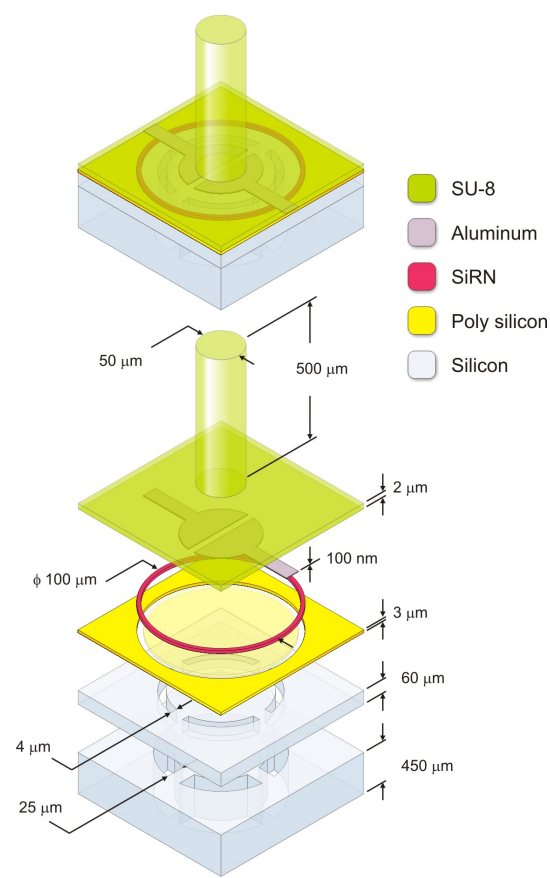

Figure 2: Top: Representation of artificial neuromast. Bottom: Sensor's layers build-up. 


\section{FABRICATION}

To fabricate the sensor we start with DSP (Double Side Polished), low resistance silicon wafers. They should be low resistance for they act as the common electrode in the sensor configuration and DSP because the backside etch process (which we will see shortly) should be uniform and optimized which is hard to achieve if started from a rough surface. Figure 3 shows a series of microscopic pictures from a single device during the fabrication process.

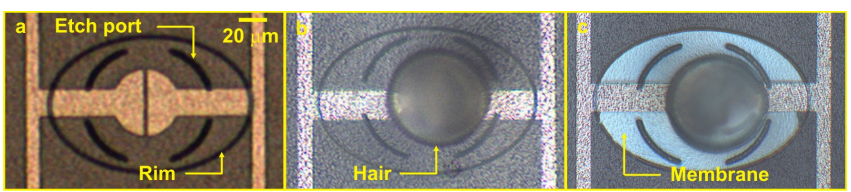

Figure 3: Microscopic pictures from the last 3 fabrication steps.

Figure 4 shows the fabrication scheme. A DRIE (Deep Reactive Ion Etching) process etches $60 \mu \mathrm{m}$ deep, $4 \mu \mathrm{m}$ wide trenches in the wafer (step a). Since these trenches are used to access the sacrificial layer from the backside and etch it, we call them "etch ports". The dimensions have been chosen to minimize the areal consumption and backside etch depth while maximizing etch species diffusion.

The next step (step b) is to grow $100 \mathrm{~nm}$ oxide and deposit $30 \mathrm{~nm}$ stoichiometric silicon nitride $\left(\mathrm{Si}_{3} \mathrm{~N}_{4}\right)$. We will point out the role of these layers later. After that, a 3 $\mu \mathrm{m}$ thick poly (or amorphous) silicon layer is deposited. This layer acts both as sacrificial layer (under the membrane area) and as a base for metal interconnects (everywhere else). It should be thick enough to fill the etch ports and smooth enough to not increase the metal interconnects' electrical resistance.

Subsequently very narrow trenches $(2 \mu \mathrm{m}$, limited by lithography facilities) are etched in the polysilicon layer to define the membrane shape (step c). These rims separate sacrificial parts from the rest of the polysilicon layer after they are filled with SiRN (Silicon Rich Nitride) in the next deposition step. To fill the rims completely it is necessary to deposit $1.2 \mu \mathrm{m}$ SiRN. Now, the whole stack of deposited materials is removed from the backside and a special DRIE process is done to reach etch ports. The selectivity of the process over silicon oxide is high such that it does not affect the $\mathrm{SiO}_{2}$ layer and therefore etch ports stick out at the bottom of the etched vias (step d).

Afterwards the wafer is oxidized to grow $1 \mu \mathrm{m}$ thick oxide at the backside (step e). In this step, the frontside SiRN layer protects the polysilicon layer from oxidation. In the etch ports, the $30 \mathrm{~nm} \mathrm{Si} \mathrm{N}_{4}$ plays the same role [5]. Having the thin oxide layer at the etch ports' sidewalls provides a good connection point for the grown oxide.

Next, using a plasma etch with high directionality the backside is bombarded to remove the protecting $\mathrm{SiO}_{2} / \mathrm{Si}_{3} \mathrm{~N}_{4}$ stack from the bottom of the etch ports and expose the sacrificial polysilicon (step f). This is followed by yet another anisotropic plasma blanket etch of $\mathrm{SiRN}$ on the frontside (step g) which should be as uniform as possible.

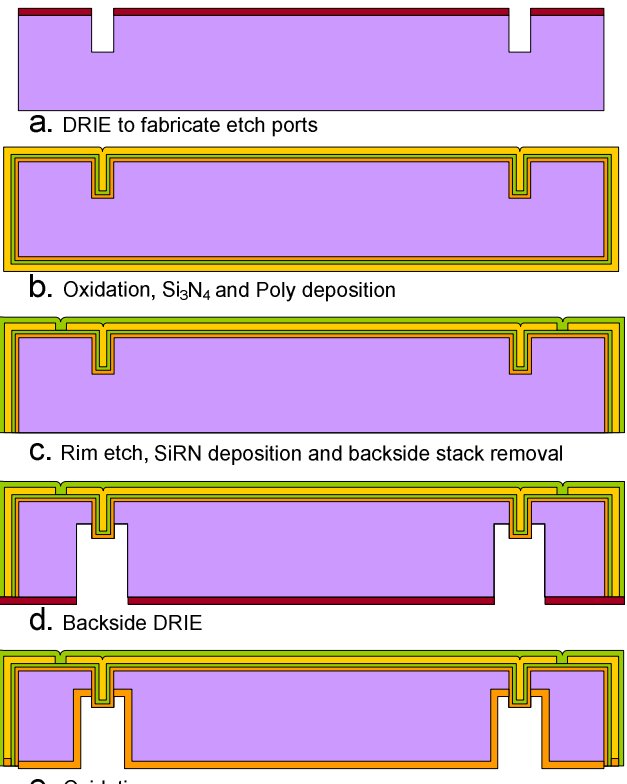
e. Oxidation
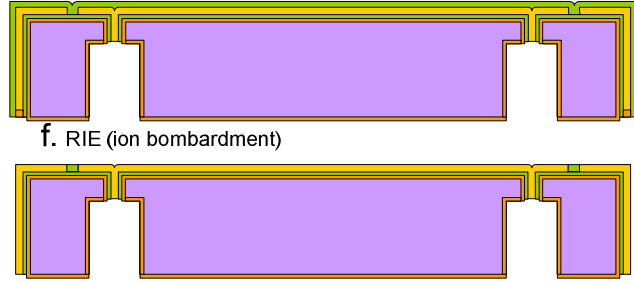

g. SiRN back etch

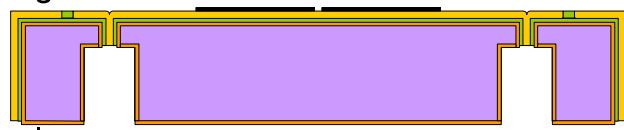

h. Aluminum electrode deposition

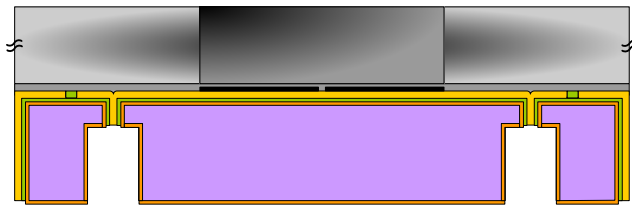

i. SU-8 spinning and exposure

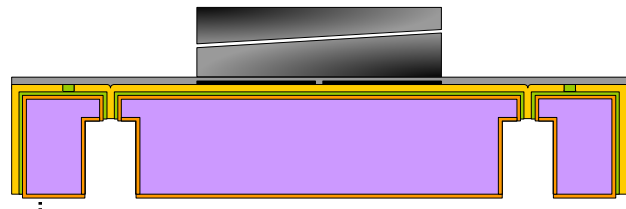

j. SU-8 developing

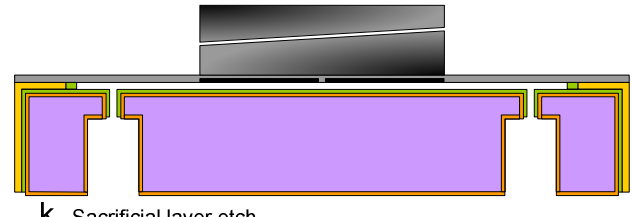

k. Sacrificial layer etch

Figure 4: Outline of the fabrication process steps

In this stage, $100 \mathrm{~nm}$ aluminum is deposited and 
patterned to form electrodes, interconnects and bond pads (step h). This deposition is done in 10 mTorr chamber pressure to increase conformality. The process continues with a thin SU-8 $2(1.8 \mu \mathrm{m}$ thick) spin coat and patterning step to form the flexible membranes. Next a thick $(\sim 500$ $\mu \mathrm{m}) \mathrm{SU}-8100$ layer is processed to realize the hairs (steps $\mathbf{i}$ and $\mathbf{j}$ ). This step can be repeated to increase the length of the hairs which results in higher drag force and mechanical sensitivity.

The last step (step k) is to etch the sacrificial layer in an isotropic plasma or $\mathrm{XeF}_{2}$. To this end the backside of the wafer is protected with the oxide grown in step e and on the frontside with the thin SU-8 2 membrane that covers the layer-stack everywhere except the bond pads.

\section{RESULTS AND DISCUSSION}

In this section we detail the fabrication steps that have been discussed in previous part, show the results and indicate the important considerations for each step. Figure 5 below shows a SEM image of the membrane cross section after step c. In this device polysilicon has been used as sacrificial layer. The thickness of this layer determines the electrode separation. To increase the electrical sensitivity we should decrease this distance but a minimum of $3 \mu \mathrm{m}$ deposition is needed to ensure complete closure of the etch ports and smooth the topography (which is important especially for the metal interconnects to follow later). However, it is possible to oxidize the sacrificial layer to some extend and remove the oxide to thin the layer while keeping the etch ports closed. This has been shown in the figure with a dotted line. Having a smooth surface helps to obtain low resistance metal lines. It is clear that in terms of uniformity and surface roughness a-Si is noticeably a better choice although the deposition rate is almost half that of polysilicon.

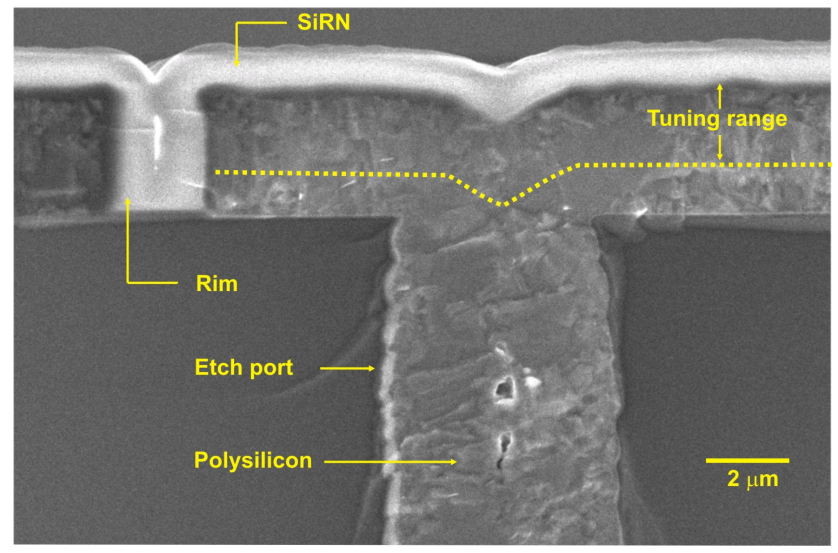

Figure 5: SEM micrograph demonstrates tuning and SiRN plug as protection rim around sacrificial layer

Figure 6 shows another SEM image of the cross section after backside etch (step e). The inset is a close up of the bottom of etch ports. The backside etch is the most critical step. Firstly, the etch process should be selective enough over silicon-oxide and/or silicon-nitride to preserve the ports. Secondly, the bottom of the etched holes should be as flat as possible otherwise, because of small misalignment, it might miss the port as indicated in Figure 7. Thirdly, the etch time and selectivity should be such that photoresist can be used as etch mask, and fourth, the uniformity of the etch rate over the entire wafer should be acceptable. This means while the etch process should be done so that ports are well exposed, over-etch can remove the protecting $\mathrm{SiO}_{2} / \mathrm{Si}_{3} \mathrm{~N}_{4}$ stack. Besides, under-etch results in closed ports and the membrane cannot be etched free.

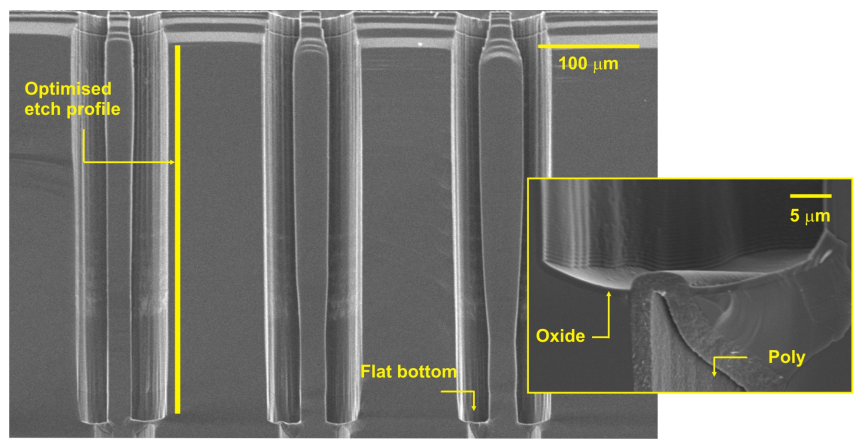

Figure 6: SEM micrograph of etch vias. The flat bottom and uniformity are important for high yield (note: wafer upside down)

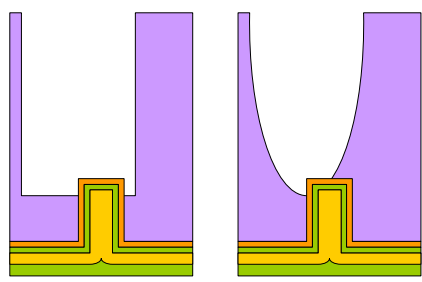

Figure 7: Detail on backside etch profile

Table 1: Backside etch recipe for Alcatel Adixen SE100

\begin{tabular}{|l|l|l|}
\hline Parameters & Etch & Deposition \\
\hline Gas & SF6 & CHF3 \\
\hline Flow (sccm) & 400 & 200 \\
\hline Time (s) & 4 & 0.5 \\
\hline Priority & 2 & 1 \\
\hline APC \% & 15 & 15 \\
\hline ICP (W) & 2500 & 2500 \\
\hline CCP(W) $[\mathrm{LF}]$ & 20 & 20 \\
\hline Pulsed (LF) ms. & 20 on/180 off & 20 on/180 off \\
\hline He (mBar) & 10 & 10 \\
\hline SH (mm) & 110 & 110 \\
\hline Temperature $\left({ }^{\circ} \mathrm{C}\right)$ & -120 & -120 \\
\hline
\end{tabular}

To fulfill these requirements, a new DRIE process for high aspect ratio etching of silicon was developed to make a flat bottom with vertical sidewalls. This new process uses $\mathrm{CHF}_{3}$ as inhibitor. Inlet holes with an aspect ratio of 22.5 are uniformly $( \pm 2 \%)$ etched over the wafer, with an etch rate of $10 \mu \mathrm{m} / \mathrm{min}$. Selectivity over $\mathrm{SiO}_{2} / \mathrm{Si}_{3} \mathrm{~N}_{4}$ stack is sufficient to stop on it: the etch rate of oxide is smaller than $10 \mathrm{~nm} / \mathrm{min}$. The etch rate for photoresist is 15 
$\mathrm{nm} / \mathrm{min}$. Sidewall profile (taper) and shape of the bottom is mainly controlled by the electrode temperature, on/off time and power of CCP (Capacitively Coupled Plasma).

In the oxidation step (step e), the thickness is determined by the selectivity of the required etch recipe for sacrificial layer etch, stress considerations and more importantly the oxidation rate of the protecting $\mathrm{SiO}_{2} / \mathrm{Si}_{3} \mathrm{~N}_{4}$ stack on the bottom of etch ports and SiRN layer on wafer front. Short oxidation times will not provide thick enough oxide layer whilst long time oxidation will oxidize the sacrificial layer. Experiments have shown that the minimum required thickness of the oxide is $1 \mu \mathrm{m}$.

Table 2 summarizes the etch recipe for opening etch ports and sacrificial layer etch (steps $\mathbf{f}$ and $\mathbf{k}$ respectively). In the anisotropic process ion bombardment will remove the $\mathrm{SiO}_{2} / \mathrm{Si}_{3} \mathrm{~N}_{4}$ protection stack and then the isotropic process will selectively remove the sacrificial polysilicon within the rim boundaries.

The next picture below is of a final device. The close up of the gap and the base of the hairs are shown in the insets. The shape of the hairs, using negative tone characteristics of $\mathrm{SU}-8$, has been tuned to increase the drag force on them [6].

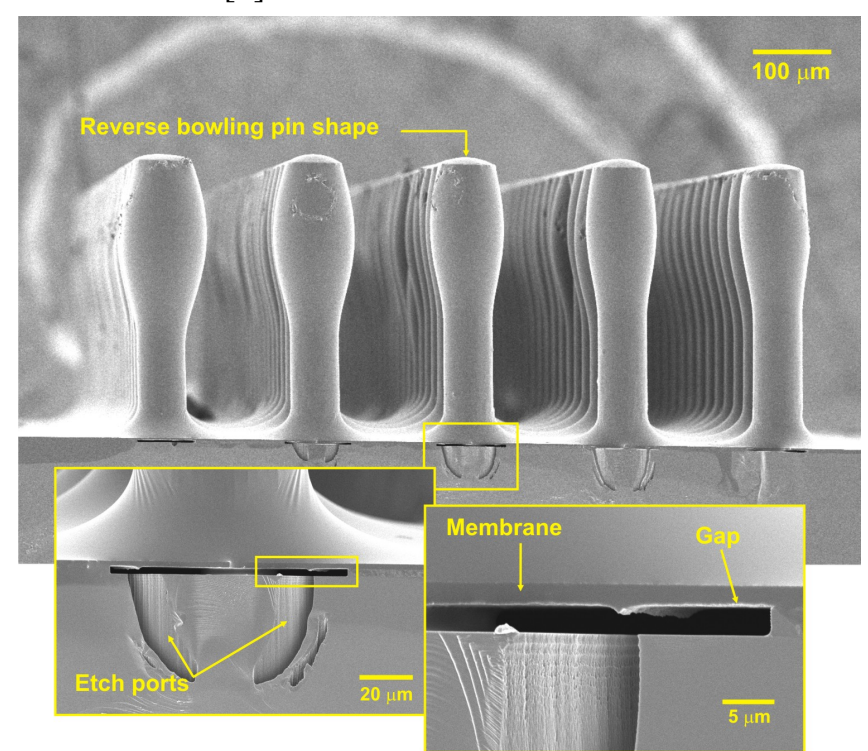

Figure 8: SEM micrograph of a sensor array. Note the bigger top of the hairs increases the drag force on them

Table 2: Etch port opening and sacrificial layer etch recipe for an Oxford Plasma Lab 100

\begin{tabular}{|l|l|l|}
\hline Parameters & Anisotropic & Isotropic \\
\hline SF6 $(\mathrm{sccm})$ & 50 & 150 \\
\hline He $(\mathrm{mTorr})$ & 20 & 20 \\
\hline ICP $(\mathrm{W})$ & 600 & 600 \\
\hline CCP $(\mathrm{W})$ & 50 & 0 \\
\hline Temperature $\left({ }^{\circ} \mathrm{C}\right)$ & 20 & 20 \\
\hline
\end{tabular}

A problem that can arise during step $\mathbf{g}$ is the sharp opening in the SiRN which fills the rim because of the keyhole (Figure 9). This opening can cause discontinuity in aluminum interconnects. To prevent this, the etch profile should be positively tapered or deposition of SiRN should be tuned not to leave a keyhole.

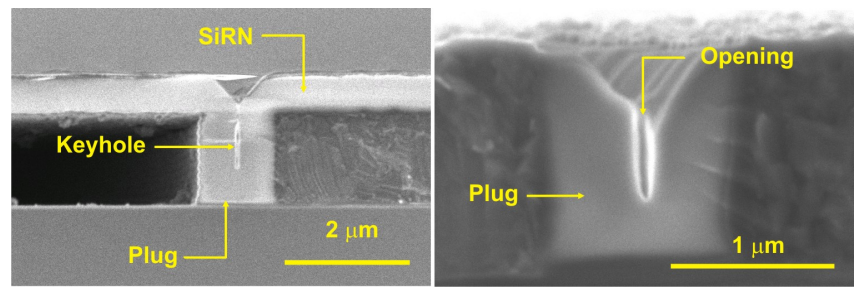

Figure 9: The keyhole in deposited SiRN in the rim can result in a sharp opening which can cause electrical discontinuity. Left: before blanket etch, right: after blanket etch

\section{CONCLUSION}

A fabrication process was presented that allowed us to successfully realize dense arrays of fully supported flexible SU-8 membranes with hairs on top. These flow sensors mimic the mechanoreceptors in lateral line of fish. Distributed sensing with closely spaced flexible membranes provides both temporally and spatially rich data. Current research focuses on characterization and optimization of the sensors.

\section{REFERENCES}

[1] S. Coombs and J.C. Montgomery, "The enigmatic lateral line system", in Comparative Hearing: Fishes and Amphibians, A. N. Popper and R. R. Fay, eds. Springer Handbook of Auditory Research, vol. 11, pp. 319-362, Springer-Verlag, N.Y., 1999.

[2] R. Voigt, A.G. Carton and J.C. Montgomery, "Responses of anterior lateral line afferent neurons to water flow", J. Experimental Biology, vol. 203, pp. 2495-2502, 2000.

[3] S. M. van Netten, "Hydrodynamic detection by cupulae in a lateral line canal: functional relations between physics and physiology", J. Biological Cybernetics, vol. 94, pp. 67-85, 2006.

[4] G. J. M. Krijnen et al., "Biomimetic micromechanical adaptive flow-sensor arrays", in Proceedings of SPIE Europe Microtechnologies for the New Millennium, vol. 6592, pp. 6592-6608, 2007.

[5] T. Enomoto, R. Ando, H. Morita and H. Nakayama, "Thermal-Oxidation Rate of a $\mathrm{Si}_{3} \mathrm{~N}_{4}$ Film and Its Masking Effect against Oxidation of Silicon", Japanese J. Applied Physics, vol. 17, pp. 1049-1058, 1978.

[6] Michael E. McConney et al., "Biologically inspired design of hydrogel-capped hair sensors for enhanced underwater flow detection", in Soft Matter, 2009.

\section{CONTACT}

* N. Izadi, tel: +31-53-489-4438; n.izadi@ewi.utwente.nl 